\title{
RESEARCH
}

\section{Effect of neighbourhood income and maternal education on birth outcomes: a population-based study}

\author{
Zhong-Cheng Luo, Russell Wilkins, Michael S. Kramer, for the Fetal and Infant Health Study Group \\ of the Canadian Perinatal Surveillance System
}

An abridged version of this article appeared in the May 9, 2006, issue of CMAJ.

\section{ABSTRACT}

Background: Maternal socioeconomic status (SES) is an important determinant of inequity in maternal and fetal health. We sought to determine the extent to which associations between adverse birth outcomes and SES can be identified using individual-level measures (maternal level of education) and community-level measures (neighbourhood income).

Methods: In Quebec, the birth registration form includes a field for the mother's years of education. Using data from birth registration certificates, we identified all births from 1991 to 2000 . Using maternal postal codes that can be linked to census enumeration areas, we determined neighbourhood income levels that reflect SES.

Results: Lower levels of both maternal education and neighbourhood income were associated with elevated crude risks of preterm birth, small-for-gestational-age (SGA) birth, stillbirth and neonatal and postneonatal death. The effects of maternal education were stronger than, and independent of, those of neighbourhood income. Compared with women in the highest neighbourhood income quintile, women in the lowest quintile were significantly more likely to have a preterm birth (adjusted odds ratio [OR] 1.14, 95\% confidence interval $[\mathrm{Cl}]$ 1.10-1.17), SGA birth (OR 1.18, 95\% Cl 1.15-1.21) or stillbirth (OR $1.30,95 \% \mathrm{Cl} 1.13-1.48$ ); compared with mothers who had completed community college or at least some university, mothers who had not completed high school were significantly more likely to have a preterm birth (adjusted OR 1.48, 95\% Cl 1.44-1.52), SGA birth (OR 1.86, $95 \% \mathrm{Cl} 1.82-1.91$ ) or stillbirth (OR 1.54, 95\% Cl 1.36-1.74).

Interpretation: Individual and, to a lesser extent, neighbourhood-level SES measures are independent indicators for subpopulations at risk of adverse birth outcomes. Women with lower education levels and those living in poorer neighbourhoods are more vulnerable to adverse birth outcomes and may benefit from heightened clinical vigilance and counselling.

Cite this article as $C M A J$ 2006;174(10). DOI:Io.1503/cmaj.051096
1 lthough reducing health inequalities is an important goal in many countries, setting priorities for and developing appropriate public health intervention programs has been a challenge, at least in part because there are insufficient data as to which subpopulations are most in need of intervention. ${ }^{1,2}$ Population-based studies of the association between socioeconomic disparities and birth outcomes can provide essential information for targeted public health programs. However, such efforts have been hampered by the paucity of data on socioeconomic status (SES) in most perinatal surveillance databases. ${ }^{3}$ For instance, US birth registrations contain data on maternal education but not on income. ${ }^{3,4}$ Canadian birth registrations are even less informative, with no data on income in any province, and data on maternal education only in Quebec and Newfoundland.,56

Intervention programs that consider at-risk subpopulations from both individual and community perspectives may help to achieve maximal benefits in reducing inequalities in maternal-fetal health. The results of recent studies suggest that measures of neighbourhood SES based on small geographic areas can be used to reflect socioeconomic gradients in health outcomes from a community perspective. ${ }^{7-10}$ Among such measures, income-related measures at the small-area level seem to be the best for revealing socioeconomic gradients. ${ }^{7-11}$ Nevertheless, it has been controversial whether the effects of area-based SES measures are attributable to differentials in individual-level SES or have some "contextual" effects beyond those explained by individual-level characteristics. There have also been no large population-based studies on the effects of neighbourhood SES on a range of birth outcomes that take into account at least some individual SES measures. To fill these gaps, we assessed the extent of socioeconomic disparities in birth outcomes by maternal education and neighbourhood income in Quebec, taking advantage of the availability of maternal education and postal codes (for geocoding small-area neighbourhoods) as recorded on birth registrations.

\section{Methods}

In this birth cohort-based study, we used Statistics Canada's updated and linked database of stillbirths, live births, and in- 
fant deaths in births to Quebec residents between I99I and 2000. The validity of the linked vital data has been well documented. ${ }^{12}$ We excluded births with missing birth weight, sex or gestational age, and those in which the gestational age was younger than 22 weeks, which left 825349 births remaining for analysis. Informed consent was not sought because the study was based on anonymous birth registration data.

Available individual-level characteristics included maternal age ( $<20,20-34, \geq 35$ years of age), education ( $<\mathrm{II}, \mathrm{II}, \mathrm{I} 2-\mathrm{I} 3$, $\geq$ I4 years of schooling), ethnicity (mother tongue as a surrogate: French, English, Aboriginal, or other), marital status (legally married, not legally married), parity (primiparous, multiparous), plurality (singleton, plural), infant sex, gestational age (in weeks) and birth weight (in grams). In the Quebec educational system, less than II years of school indicates the person has not graduated from high school, II years indicates high school graduation, I2-I3 years some community college (CEGEP vocational or pre-university studies), and I4 years or more graduation from community college or at least some years at university. This categorization of maternal education has been strongly associated with infant mortality in Quebec. ${ }^{13}$

We assigned each birth to the corresponding census enumeration area and census metropolitan area or census agglomeration (CMACA) through geocoding based on the postal code of the mother's place of residence as reported on the birth registration. ${ }^{14}$ An enumeration area typically consists of $125-440$ dwellings of relatively homogenous socioeconomic status. ${ }^{15}$ For the $200 \mathrm{I}$ census, the analysis was based on dissemination areas, which replaced enumeration areas as the smallest unit of geography for which census data are disseminated. For simplicity, we refer to both as enumeration areas or "neighbourhoods." CMACAs are economic communities of 10 ooo or more people that include adjacent districts with high commuting flows into the central area. For example, the Montreal census metropolitan area includes not only the entire Island of Montreal, but also all of Laval, the commuting areas north of Laval and the South Shore suburbs. Rural and urban areas were defined according to Statistics Canada's recommended definition: all CMACAs with a population of 10 000 or more (I996 census) were urban, and the remaining areas were rural. ${ }^{16}$ Analyses were done for all neighbourhoods, and for rural and urban areas separately.

We created neighbourhood income quintiles to reflect the relative SES of the neighbourhood in which the mother lived as compared with other neighbourhoods within the same CMACA. ${ }^{11}$ A household-size-adjusted "income per single person equivalent" was first calculated for each enumeration area, using the Canadian census data from the closest census year (I99I, I996 or 200I). Enumeration areas within the same CMACA were then ranked into quintiles from the richest (QI)

Table 1: Maternal characteristics by neighbourhood income quintile in Quebec, 1991-2000

\begin{tabular}{|c|c|c|c|c|c|c|}
\hline \multirow[b]{2}{*}{ Characteristic } & \multicolumn{5}{|c|}{ Births by neighbourhood income quintile, * \% } & \multirow[b]{2}{*}{$\begin{array}{c}\text { Total } \\
n=825349\end{array}$} \\
\hline & $\begin{array}{l}\text { Q1 (richest) } \\
n=148354\end{array}$ & $\begin{array}{c}\text { Q2 } \\
n=163369\end{array}$ & $\begin{array}{c}\text { Q3 } \\
n=164192\end{array}$ & $\begin{array}{c}Q 4 \\
n=167509\end{array}$ & $\begin{array}{l}\text { Q5 (poorest) } \\
n=181925\end{array}$ & \\
\hline \multicolumn{7}{|l|}{ Age, yr } \\
\hline$<20$ & 2.3 & 2.9 & 3.8 & 5.2 & 7.9 & 37343 \\
\hline $20-34$ & 82.4 & 86.0 & 86.1 & 85.4 & 82.3 & 696368 \\
\hline \multicolumn{7}{|l|}{ Education, yr† } \\
\hline$<11$ & 7.0 & 9.3 & 12.4 & 16.5 & 25.9 & 117694 \\
\hline 11 & 7.8 & 10.6 & 12.4 & 13.9 & 14.7 & 96727 \\
\hline $12-13$ & 22.1 & 27.3 & 29.1 & 29.2 & 27.2 & 218458 \\
\hline$\geq 14$ & 63.1 & 52.8 & 46.1 & 40.5 & 32.2 & 372483 \\
\hline \multicolumn{7}{|c|}{ Ethnicity by mother tongue } \\
\hline French & 78.3 & 84.2 & 84.3 & 80.5 & 70.0 & 650567 \\
\hline English & 12.7 & 7.8 & 6.6 & 7.0 & 6.8 & 66065 \\
\hline Aboriginal & 0.8 & 0.5 & 0.4 & 0.7 & 1.9 & 7394 \\
\hline Other & 8.2 & 7.5 & 8.7 & 11.9 & 21.4 & 96655 \\
\hline \multicolumn{7}{|l|}{ Parity } \\
\hline Primiparous & 41.9 & 43.0 & 43.8 & 45.8 & 45.6 & 363816 \\
\hline Multiparous & 58.1 & 57.0 & 56.2 & 54.2 & 54.4 & 461315 \\
\hline
\end{tabular}

${ }^{*} \chi^{2}$ tests for differences in any characteristic among the 5 neighbourhood income quintiles were all significant at $p<0.001$.

†In Quebec, 11 years of education indicates high school graduation, 12-13 years indicates some community college, and 14 years or more indicates graduation from community college or at least some years at a university. 
to the poorest (Q5) according to income per single person equivalent. Each quintile contained about one-fifth of the total population within each CMACA (but not necessarily onefifth of all births). Creating the quintiles within each CMACA helped take account of inter-regional differences in housing costs. A neighbourhood income quintile was assigned to each birth based on the enumeration area of the mother's place of residence.

Main outcomes were preterm birth $(<37$ completed weeks in gestational age), small-for-gestational-age birth (SGA, < roth percentile, based on a recent Canadian fetal growth standard), ${ }^{17}$ stillbirth, neonatal death (0-27 days) and postneonatal death (28-364 days). Causes of infant death were grouped according to the classification of the International Collaborative Effort on Perinatal and Infant Mortality, ${ }^{18}$ using International Classification of Diseases (ICD)9 codes for births in I99I-I999 or ICD-Io codes for births in 2000. Categories included congenital conditions, immaturity-related conditions, asphyxia, sudden infant death syndrome (SIDS), infection, external causes, other specific conditions, and remaining causes. The risk of each outcome was assessed for both neighbourhood income quintile and maternal education. Crude and adjusted odds ratios (ORs) with 95\% confidence intervals (CIs) were compared with assess the potential confounding effects of other variables. Ad- justed ORs were estimated using both ordinary (single-level) and multilevel logistic regression analyses. Since identical effect estimates were obtained (very low intracluster variations), we therefore present the adjusted ORs based on ordinary logistic regression analysis. Interactions between variables were checked, and no significant influences on effect estimates of interest were observed.

\section{Results}

Substantial differences in maternal characteristics were observed across neighbourhood income quintiles (Table I). Mothers living in poorer neighbourhoods were much more likely to be unmarried, younger than 20 years of age, and to not have graduated from high school, and they were much less likely to be 35 years of age or older. The proportion of women with English as their mother tongue was much higher in the richest quintile ( $13 \%$ ) than in other neighbourhood income quintiles ( $7 \%-8 \%)$.

Increasingly higher rates of preterm birth, SGA, stillbirth, neonatal death and postneonatal death were observed among mothers with lower levels of education and across neighbourhood income quintiles from the richest to the poorest (Table 2). The risk gradients for adverse birth outcomes were larger across the maternal education strata.

Table 2: Adverse birth outcomes with crude and adjusted odds ratios (ORs) by neighbourhood income quintile (Q) and maternal education in Quebec, 1991-2000

\begin{tabular}{|c|c|c|c|c|c|}
\hline $\begin{array}{l}\text { Income quintile } \\
\text { and maternal } \\
\text { education level }\end{array}$ & Preterm births, \% & SGA births, \% & $\begin{array}{l}\text { Stillbirths, } \\
\text { no. per } 1000\end{array}$ & $\begin{array}{l}\text { Neonatal deaths, } \\
\text { no. per } 1000\end{array}$ & $\begin{array}{c}\text { Postneonatal deaths, } \\
\text { no. per } 1000\end{array}$ \\
\hline \multicolumn{6}{|c|}{ Neighbourhood income quintile } \\
\hline Q1 (richest) & 6.7 & 9.1 & 3.2 & 3.3 & 1.4 \\
\hline Q2 & 7.0 & 9.8 & 3.4 & 2.9 & 1.4 \\
\hline Q3 & 7.2 & 10.6 & 3.9 & 3.3 & 1.3 \\
\hline Q4 & 7.4 & 11.2 & 4.0 & 3.8 & 1.5 \\
\hline Q5 (poorest) & 8.2 & 12.3 & 4.6 & 3.9 & 2.0 \\
\hline$p$ value for trend ${ }^{*}$ & $<0.001$ & $<0.001$ & $<0.001$ & $<0.001$ & $<0.001$ \\
\hline \multicolumn{6}{|l|}{ Q5 v. Q1 } \\
\hline Crude OR $(95 \% \mathrm{CI})$ & $1.23(1.20-1.26)$ & $1.40(1.37-1.43)$ & $1.44(1.29-1.62)$ & $1.16(1.03-1.30)$ & $1.48(1.25-1.75)$ \\
\hline Adjusted OR $\neq(95 \% \mathrm{Cl})$ & $1.14(1.10-1.17)$ & $1.18(1.15-1.21)$ & $1.30(1.13-1.48)$ & $1.03(0.90-1.17)$ & $1.08(0.89-1.30)$ \\
\hline \multicolumn{6}{|l|}{ Maternal education, yr† } \\
\hline$\geq 14$ & 6.3 & 8.5 & 2.5 & 2.8 & 1.1 \\
\hline $12-13$ & 7.3 & 11.3 & 3.0 & 3.4 & 1.4 \\
\hline 11 & 8.0 & 12.5 & 3.1 & 3.7 & 1.8 \\
\hline$<11$ & 8.9 & 14.4 & 3.8 & 3.9 & 2.8 \\
\hline$p$ value for trend ${ }^{*}$ & $<0.001$ & $<0.001$ & $<0.001$ & $<0.001$ & $<0.001$ \\
\hline \multicolumn{6}{|l|}{$<11 \mathrm{v} . \geq 14 \mathrm{yr}$} \\
\hline Crude OR (95\% CI) & $1.46(1.42-1.49)$ & $1.80(1.77-1.84)$ & $1.53(1.37-1.72)$ & $1.41(1.26-1.57)$ & $2.64(2.27-3.06)$ \\
\hline Adjusted OR $\neq(95 \% \mathrm{Cl})$ & $1.48(1.44-1.52)$ & $1.86(1.82-1.91)$ & $1.54(1.36-1.74)$ & $1.36(1.20-1.54)$ & $2.03(1.72-2.41)$ \\
\hline
\end{tabular}

Note: $\mathrm{SGA}=$ Small for gestational age, $\mathrm{Cl}$ = confidence interval.

*Cochran-Armitage tests for trends across strata by maternal education or neighbourhood income.

tIn Quebec, 11 years of education indicates high school graduation, 12-13 years indicates some community college, and 14 years or more indicates graduation from community college or at least some years at a university.

$\ddagger$ Adjusted ORs in logistic regression included the following variables: infant sex, parity, plurality and maternal ethnicity (mother tongue), age, education level, marital status, and neighbourhood income quintile. 
After controlling for maternal education and other individual characteristics, the adjusted ORs for adverse birth outcomes comparing the poorest and richest neighbourhood income quintiles were moderately lower than the crude ORs and the adjusted ORs became nonsignificant for neonatal and postneonatal death (Table 2). By contrast, after controlling for neighbourhood income and other individual characteristics, the adjusted ORs of adverse birth outcomes comparing mothers who had not completed high school with mothers who had completed community college or some university were virtually identical to the crude ORs for all birth outcomes except postneonatal death. The largest ORs across the maternal education categories were observed for SGA and postneonatal death.

Stepwise-adjusted risk analyses (not shown) revealed that, for any adverse birth outcome, maternal education was the only individual-level factor having a greater than $5 \%$ impact on the effect size estimates for neighbourhood income quintile. In contrast, neighbourhood income quintile had virtually no impact on the effect size estimates for maternal education.

In patterns similar to those for the crude risks in Table 2, the adjusted ORs (results available upon request) showed clear risk gradients across the maternal education strata for all adverse birth outcomes. The etiologic fractions (population attributable risks, based on the adjusted ORs) for maternal education (all 3 strata, using $\geq \mathrm{I} 4$ years as the reference) were $13 \%$ for preterm birth, $21 \%$ for SGA, $15 \%$ for stillbirth, I2 $\%$ for neonatal death and $22 \%$ for postneonatal death. By contrast, the adjusted ORs showed a smaller risk gradient across neighbourhood income quintiles. The etiologic fractions for neighbourhood income (all 4 strata, using the richest quintile as the reference) were $5 \%$ for preterm birth, $7 \%$ for SGA and $\mathrm{I} 2 \%$ for stillbirth.

To understand the causes of the differences in neonatal and postnatal mortality across neighbourhood income quintiles, we further examined rates of death by gestational age and by cause of death (Table 3). The risk of neonatal death by gestational age was not significantly different across neighbourhood income quintiles. The risk of postneonatal death by gestational age was higher for the poorest neighbourhood income quintile compared with that for the highest income quintile at all gestational ages except 28-3I weeks, but the difference was significant for term births only. A significantly elevated risk of neonatal death due to immaturity-related conditions (OR I.29), and of postneonatal death due to SIDS (OR I.63), was observed when births in the poorest and richest neighbourhood income quintiles were compared. Other causes of death were not significantly different across neighbourhood income quintiles.

Fig. I shows the adjusted ORs of adverse birth outcomes associated with maternal education and neighbourhood income in rural and urban areas separately (detailed results are provided in Table 4). The adjusted ORs were obtained from logistic regression models including infant sex, parity, plurality, maternal age, education, ethnicity, marital status and neighbourhood income quintile. The adjusted ORs comparing mothers in the poorest and richest neighbourhood income quintiles were somewhat lower than crude ORs (not

Table 3: Rates of gestational age-specific neonatal and postneonatal death and of cause-specific infant death by neighbourhood income quintile in Quebec, 1991-2000

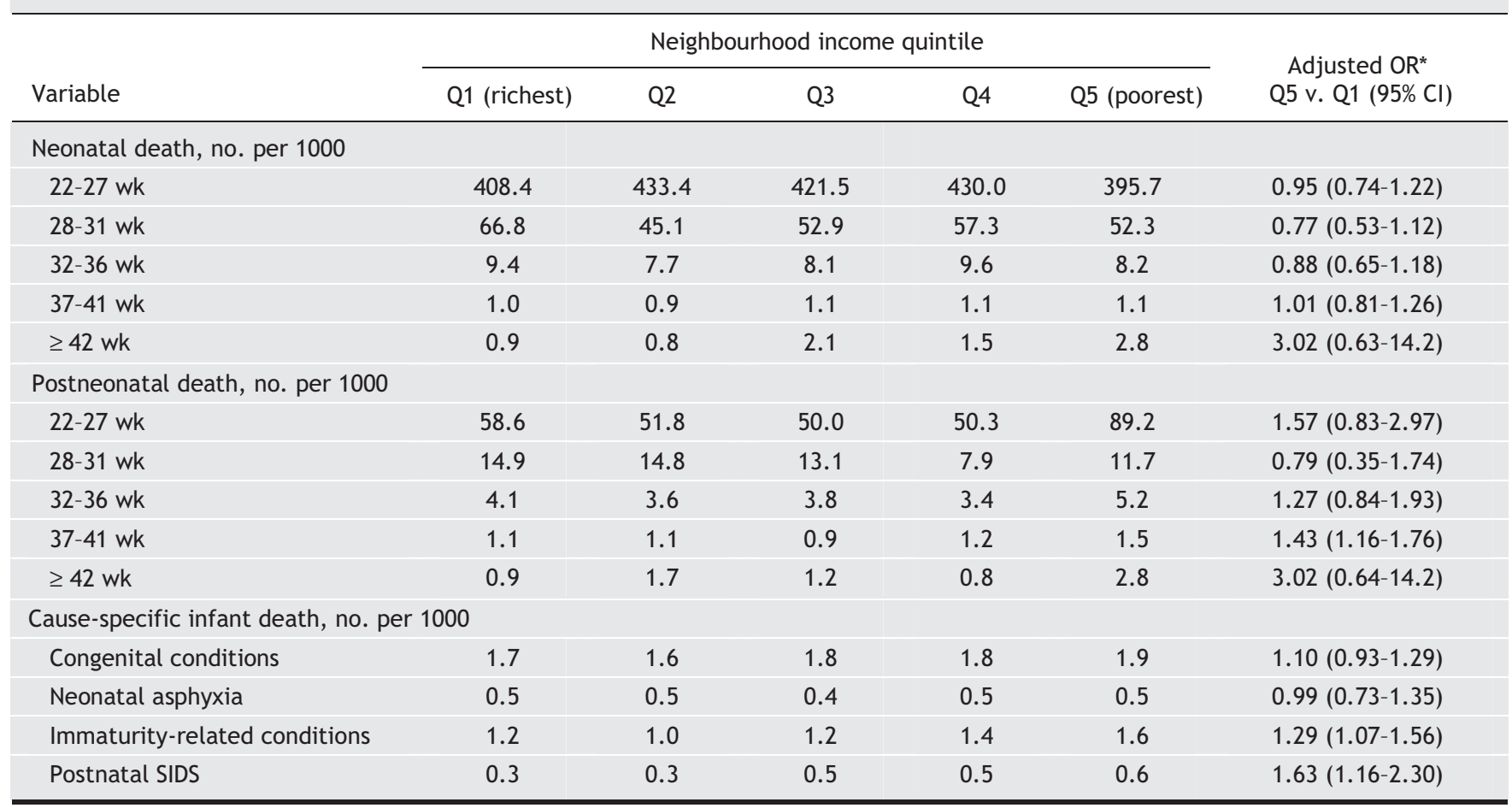

Note: $\mathrm{OR}=$ odds ratio, $\mathrm{Cl}=$ confidence interval, SIDS = sudden infant death syndrome.

*Adjusted ORs from logistic regression included the following variables: infant sex, parity, plurality, and maternal age, education, ethnicity (mother tongue), marital status, and neighbourhood income quintile. 
shown) and showed modest but significantly elevated risks of preterm birth, SGA, stillbirth and neonatal death in urban but not rural areas. Adjusted ORs for cause-specific neonatal and postneonatal death across neighbourhood income quintiles were all nonsignificant (not shown). In urban areas, a large and significant crude OR for death due to SIDS (OR 2.02, 95\% CI I.32-3.I0) was observed among mothers in the poorest neighbourhood income quintile compared with those in the richest income quintile, but the corresponding adjusted OR was nonsignificant.

In both rural and urban areas, the adjusted ORs for maternal education showed moderately and significantly elevated risks of preterm birth, SGA, stillbirth, neonatal death and postneonatal death for mothers who had not completed high school compared with mothers who had completed community college or some university. The adjusted ORs for maternal education were slightly higher in urban areas compared with rural areas for all outcomes except for neonatal death. Analyses of the risks of cause-specific infant death associated with low maternal education revealed a modestly elevated risk of infant death due to congenital conditions in urban but not rural areas (adjusted OR I.53, 95\% CI I.26-I.84), a significantly elevated risk of infant death due to immaturity-related conditions (adjusted OR 2.26, 95\% CI I. 26-I.84 in rural areas V. I. $35,95 \%$ CI I.07-I.69 in urban areas), and a more substantially elevated risk of infant death due to SIDS (adjusted OR 2.69, 95\% CI I.49-4.88 in rural areas v. 2.70, 95\% CI I.87-3.9I in urban areas) in both rural and urban areas. The elevated risk of infant death due to immaturity-related conditions associated with low maternal education was modestly higher in rural areas.

\section{Interpretation}

The results of some studies have suggested that the effects of neighbourhood-based measures of SES on birth outcomes may be independent of individual-level measures of SES. ${ }^{19-21}$ Our study is the first large population-based study to demonstrate a higher risk of multiple adverse birth outcomes in poorer neighbourhoods that is at least in part independent of large differences in an individual SES measure (maternal education) and other observed characteristics. We further demonstrate that the elevated adjusted risks in poorer neighbourhoods occurred almost exclusively in urban areas, which account for about $75 \%$ of all births in Canada. Among individual measures of SES, education is considered the most powerful determinant of health. ${ }^{22}$ In our study, the effects of maternal education were clearly larger than, and independent of, the effects of neighbourhood income. Moreover, the population health impact of maternal education was stronger than that of neighbourhood income, as indicated by their respective etiologic fractions. In both urban and rural areas, the effects of low maternal education levels were strongest for SGA and postneonatal death. We also found that infants of mothers with low education levels in rural areas seemed more vulnerable to neonatal death, particularly for death due to immaturity-related conditions. We speculate that access to highquality neonatal intensive care may be more limited for mothers with low education levels who live in a rural setting.

A small but growing literature has revealed disparities in birth outcomes by neighbourhood SES..$^{9-11,19-21}$ The neighbourhood disparities in birth outcomes observed here were smaller than those reported in the United States. For example, we observed a crude OR of I.38 (95\% CI I.34-I.4I) for low birth weight (results not shown, since SGA is a better fetal growth indicator), which is substantially lower than the 
Table 4: Crude and adjusted odds ratios (ORs) of adverse birth outcomes by neighbourhood income quintile and maternal education in rural and urban areas of Quebec, 1991-2000

\begin{tabular}{|c|c|c|c|c|}
\hline \multirow[b]{2}{*}{ Outcome } & \multicolumn{2}{|c|}{ Rural$^{*}(n=176$ 496) } & \multicolumn{2}{|c|}{$\operatorname{Urban}^{*}(n=648853)$} \\
\hline & Crude OR $(95 \% \mathrm{Cl})$ & Adjusted OR† (95\% CI) & Crude OR $(95 \% \mathrm{Cl})$ & Adjusted OR† $(95 \% \mathrm{Cl})$ \\
\hline \multicolumn{5}{|c|}{ Neighbourhood income: Poorest v. richest quintile } \\
\hline Preterm birth & $1.05(0.99-1.10)$ & $1.01(0.95-1.10)$ & $1.29(1.25-1.33)$ & $1.16(1.12-1.20)$ \\
\hline SGA & $1.08(1.03-1.13)$ & $1.00(0.95-1.05)$ & $1.50(1.47-1.54)$ & $1.24(1.20-1.27)$ \\
\hline Postneonatal death & $1.13(.082-1.55)$ & $0.94(0.67-1.32)$ & $1.68(1.37-2.06)$ & $1.20(0.96-1.50)$ \\
\hline \multicolumn{5}{|l|}{ Cause-specific infant death } \\
\hline Congenital conditions & $0.91(0.64,1.29)$ & $0.84(0.59,1.21)$ & $1.16(0.96-1.390$ & $1.07(0.88-1.31)$ \\
\hline Asphyxia & $0.48(0.22-1.01)$ & $0.56(0.28-1.15)$ & $1.16(0.82-1.65)$ & $1.03(0.70-1.52)$ \\
\hline SGA & $1.66(1.59-1.73)$ & $1.77(1.69-1.85)$ & $1.85(1.81-1.90)$ & $1.82(1.77-1.87)$ \\
\hline Stillbirth & $1.44(1.16-1.80)$ & $1.27(1.00,1.62)$ & $1.55(1.36,1.77)$ & $1.65(1.43-1.90)$ \\
\hline Neonatal death & $1.39(1.12-1.74)$ & $1.56(1.24-1.95)$ & $1.38(1.21,1.58)$ & $1.27(1.11-1.47)$ \\
\hline Postneonatal death & $2.49(1.86-3.32)$ & $2.07(1.51-2.84)$ & $2.61(2.19-3.10)$ & $2.14(1.76-2.60)$ \\
\hline \multicolumn{5}{|l|}{ Cause-specific infant death } \\
\hline Congenital conditions & $1.22(0.92-1.63)$ & $1.21(0.90-1.63)$ & $1.48(1.24-1.77)$ & $1.53(1.26-1.84)$ \\
\hline Asphyxia & $1.22(0.61-2.22)$ & $1.57(0.84-2.94)$ & $1.35(0.96-1.90)$ & $1.23(0.85-1.78)$ \\
\hline Immaturity-related conditions & $1.90(1.33-2.72)$ & $2.26(1.56-3.26)$ & $1.50(1.21-1.85)$ & 1.35 (1.07-1.69) \\
\hline SIDS & $4.42(2.42-8.06)$ & $2.69(1.49-4.88)$ & $5.13(3.60-7.30)$ & $2.70(1.87-3.91)$ \\
\hline
\end{tabular}

Note: $\mathrm{SGA}=$ small for gestational age, $\mathrm{CI}=$ confidence interval, SIDS = sudden infant death syndrome.

*Urban areas are defined as having a population $\geq 10000$; areas with a population $<10000$ are rural.

†Adjusted ORs from logistic regression included the following variables: infant sex, parity, plurality, and maternal age, education, ethnicity (by mother tongue), marital status, and neighbourhood income quintile.

fln Quebec, $<11$ years of education indicates not having graduated from high school, and $\geq 14$ years indicates graduation from community college or at least some years at a university.

crude OR of 2.06 (95\% CI I.93-2.I9) for low birth weight reported recently following comparison of the poorest and richest neighbourhood income quintiles based on census block groups (a census geographic unit comparable to a Canadian census enumeration area) in Massachusetts and Rhode Island. ${ }^{9}$ Greater neighbourhood segregation in the United States may explain the larger disparities in health outcomes compared with those observed in previous studies. ${ }^{23-25}$ Nevertheless, we found socioeconomic disparities in a number of birth outcomes at both the individual (maternal education) and community (neighbourhood income) levels; studies with information on individual or neighbourhood SES measures only (as happens in many studies) may underestimate or distort the true extent of such socioeconomic disparities in birth outcomes.

The effect estimates for neighbourhood income quintile in rural areas may have been attenuated because of lesser precision of rural postal codes in determining enumeration areas (nondifferential misclassification of exposure). Canadian perinatal data do not have information on many potential confounders or effect mediators, including family income, maternal occupation, smoking and others. Differences in other unmeasured individual-level SES measures and risk factors may explain or mediate the poor birth outcomes in poorer neighbourhoods.

The impact of neighbourhood income is evident for stillbirth, preterm birth and the risk of SGA. These findings suggest that women from poor neighbourhoods may warrant increased vigilance from perinatal care providers. The large beneficial impact of higher maternal education on postneonatal mortality may operate through better knowledge of infant care, especially sleep position, ${ }^{26}$ as evidenced by the large differences in SIDS risk. Physicians, midwives and public health nurses should be vigilant in referring mothers with low education levels and high-risk antenatal conditions to higher levels of care, and mothers with low education levels should receive counselling on appropriate infant care practices. The results of our study also suggest that women from poor neighbourhoods and those with low education levels may benefit from heightened clinical vigilance and coun- 
selling during perinatal period. Further studies are needed to understand the factors mediating these higher risks at both individual and community levels, so that effective intervention programs to reduce socioeconomic inequalities in perinatal health can be implemented.

This article has been peer reviewed.

From the departments of Epidemiology and Biostatistics and of Pediatrics (Luo, Kramer), McGill University, and the Department of Obstetrics and Gynecology (Luo), Sainte Justine Hospital, University of Montreal, Montréal, Qué., and the Health Analysis and Measurement Group, Statistics Canada and the Department of Epidemiology and Community Medicine (Wilkins), University of Ottawa, Ottawa, Ont.

Competing interests: None declared.

Contributors: All of the authors contributed substantially to the study conception and design and to the interpretation of data. Zhong-Cheng Luo and Russell Wilkins conducted the analyses. Zhong-Cheng Luo was responsible for the literature review and for writing the manuscript. Michael Kramer and Russell Wilkins revised the article critically for important intellectual content. All of the authors approved the version to be published.

Acknowledgements: We are grateful to the valuable comments on a preliminary draft of the manuscript from members of the Fetal and Infant Health Study Group of the Canadian Perinatal Surveillance System. Michael Kramer is supported by a Senior Investigator Award from the Canadian Institutes of Health Research (CIHR). Zhong-Cheng Luo is supported by a postdoctoral fellowship (2002-2004) from CIHR, a Junior Scholar Award from Sainte Justine Hospital (2004-2005) and the Quebec Foundation for Health Research (2005-). We are grateful to the Quebec Vital Statistics Registrar and Statistics Canada for providing access to their data.

\section{REFERENCES}

I. Bhutta ZA, Darmstadt GL, Hasan BS, et al. Community-based interventions for improving perinatal and neonatal health outcomes in developing countries: a review of the evidence. Pediatrics 2005;II5(Suppl 2):519-6I7.

2. Health Canada. A proactive approach to good health. Available: www.oagbvg.gc.ca/domino/reports.nsf/html/orogce.html (accessed 2006 Feb 23).

3. Krieger N, Chen JT, Ebel G. Can we monitor socioeconomic inequalities in health? A survey of U.S. health departments' data collection and reporting practices. Public Health Rep I997; II2:48I-9I.

4. Dubay L, Joyce T, Kaestner R, et al. Changes in prenatal care timing and low birth weight by race and socioeconomic status: implications for the Medicaid expansions for pregnant women. Health Serv Res 200I;36:373-98.

5. Health Canada. Canadian Perinatal Health Report, 2003. Ottawa: Public Health Agency of Canada; 2003

6. Luo ZC, Wilkins R, Platt RW, For the Fetal and Infant Health Study Group of the Canadian Perinatal Surveillance System. Risks of adverse pregnancy outcomes among Inuit and North American Indian women in Quebec. Paediatr Perinat Epidemiol 2004;18:40-50.
7. Krieger N, Chen JT, Waterman PD, et al. Geocoding and monitoring of US socioeconomic inequalities in mortality and cancer incidence: does the choice of areabased measure and geographic level matter? The Public Health Disparities Geocoding Project. Am J Epidemiol 2002;156:47I-82.

8. Krieger N, Chen JT, Waterman PD, et al. Race/ethnicity, gender, and monitoring socioeconomic gradients in health: a comparison of area-based socioeconomic measures-the public health disparities geocoding project. Am J Public Health 2003;93:1655-7I.

9. Krieger N, Chen JT, Waterman PD, et al. Choosing area based socioeconomic measures to monitor social inequalities in low birth weight and childhood lead poisoning: The Public Health Disparities Geocoding Project (US). J Epidemiol Community Health 2003;57:186-99.

Io. Trends in mortality by neighbourhood income in urban Canada from I971 to Ig96. Ottawa: Statistics Canada; 2002. Cat no 82-003-SIE.

II. Luo ZC, Kierans WJ, Wilkins R, et al.; British Columbia Vital Statistics Agency. Temporal trends in disparities in birth outcomes by neighborhood income in rural vs urban British Columbia. Epidemiology 2004;15:679-86.

I2. Fair M, Cyr M, Allen AC, et al.; Fetal and Infant Health Study Group. An assessment of the validity of a computer system for probabilistic record linkage of birth and infant death records in Canada. Chronic Dis Can 2000;21:8-13.

I3. Chen J, Fair M, Wilkins R. Cyr M and the Fetal and Infant Mortality Study Group of the Canadian Perinatal Surveillance System. Maternal education and fetal and infant mortality in Quebec. Health Rep i998;10:53-64.

I4. Wilkins R. Automated geographic coding based on the Statistics Canada postal code conversion files. Ottawa: Statistics Canada; 200I. Cat. no. 82Foo86-XDB.

I5. I9g6 Census dictionary. Final ed. Ottawa: Statistics Canada; I999. Cat. no. 92-30IE.

I6. Du Plessis V, Beshiri R. Bollman RD, et al. Rural and Small Town Canada Analysis Bulletin Vol. 3, no. 3 (November 200I). Ottawa: Statistics Canada; 200I. Cat. no. 2I-006-XIE.

I7. Kramer MS, Platt RW, Wen SW, et al. A new and improved population-based Canadian Reference for birth weight for gestational age. Pediatrics 2001;108:E35.

I8. Cole S, Hartford RB, Bergsjo P, et al. International collaborative effort (ICE) on birthweight, plurality, perinatal, and infant mortality. Acta Obstet Gynecol Scand I989;68:113-7.

I9. Pickett KE, Ahern JE, Selvin S, et al. Neighborhood socioeconomic status, maternal race and preterm delivery: a case-control study. Ann Epidemiol 2002;12:410-8.

20. O'Campo P, Xue X, Wang MC, et al. Neighborhood risk factors for low birthweight in Baltimore: a multilevel analysis. Am J Public Health 1997;87:1113-8.

2I. Pearl M, Braveman P, Abrams B. The relationship of neighborhood socioeconomic characteristics to birthweight among 5 ethnic groups in California. Am J Public Health 200I;91:I808-I4.

22. Winkleby MA, Jatulis DE, Frank E, et al. Socioeconomic status and health: how education, income, and occupation contribute to risk factors for cardiovascular disease. Am J Public Health I992;82:816-20.

23. Lynch JW, Kaplan GA, Pamuk ER, et al. Income inequality and mortality in metropolitan areas of the United States. Am J Public Health 1998;88:1074-80.

24. Ross NA, Wolfson MC, Dunn JR, et al. Relation between income inequality and mortality in Canada and in the United States: cross sectional assessment using census data and vital statistics. BMJ 2000;320:898-902.

25. Lynch J, Smith GD, Hillemeier M, et al. Income inequality, the psychosocial environment, and health: comparisons of wealthy nations. Lancet 2001;358:194-200.

26. American Academy of Pediatrics AAP Task Force on Infant Positioning and SIDS Positioning and SIDS. Pediatrics 1992;89:1120-6.

Correspondence to: Dr. Zhong-Cheng Luo, Bureau 4986, Obstetrics and Gynecology, Sainte-Justine Hospital, 3175 chemin de la Côte-Ste-Catherine, Montréal QC $\mathrm{H}_{3} \mathrm{~T}_{1} \mathrm{C}_{5}$; zhong-cheng.luo@recherche-ste-justine.qc.ca 